\title{
Un algoritmo en el entorno del software $R$ para modelar el rendimiento de materia seca en pastos con
} fertilización nitrogenada

\section{An algorithm in the $\mathrm{R}$ environment to model the dry matter yield in pastures with nitrogened fertilization}

\author{
Danny A. Villegas ${ }^{1}$, Nora Valbuena ${ }^{1}$ y Manuel E. Milla ${ }^{2 *}$
}

\section{RESUMEN}

El presente trabajo tiene como objetivo modelar el comportamiento productivo de pastos, específicamente Brachiaria brizantha $\mathrm{cv}$. Toledo bajo fertilización nitrogenada mediante la utilización de algoritmos en el entorno de programación del software $\mathrm{R}$, con el fin de estimar la producción de materia seca a diferentes edades de corte. Para lo cual se consideró un modelo no lineal como lo es el logístico. Se evidenció la utilidad del software $\mathrm{R}$ en la construcción de bandas de confianza sobre el ajuste de modelos no lineales, específicamente el logístico para la estimación de la producción de materia seca en pastos bajo condiciones de fertilización nitrogenada a diferentes edades de corte. El modelo logístico mostró una alta precisión para explicar los $\mathrm{kg} \mathrm{MS} \cdot \mathrm{ha}^{-1} \mathrm{de} B$. brizantha $\mathrm{cv}$. Toledo acumulados en función de la edad de corte y de los niveles de fertilización nitrogenada, así como la presencia de un posible punto de inflexión, el cual en la época de lluvia se ubicó alrededor del día 100 y en la época seca alrededor del día 40, asociado a la transición entre una época y otra. Finalmente, los resultados sugieren que la especie forrajera $B$. brizantha está influenciada por la dosis de fertilización, edad de corte, época del año y las condiciones edafoclimáticas.

Palabras claves: Simulación, script, cultivos perennes, modelos no lineales.

\begin{abstract}
In this paper the productive behavior of pastures was simulated, specifically Brachiaria brizantha cv. Toledo under nitrogen fertilization by the use of algorithms in the R software programming environment, in order to estimate dry matter production at different cutting ages. For which a non-linear (logistic) model was considered. The usefulness of software $\mathrm{R}$ in the construction of confidence bands on the adjustment of nonlinear models, specifically logistic for the estimation of dry matter production in pastures under nitrogen fertilization conditions at different cutting ages was evidenced. The logistic model showed a high precision to explain the $\mathrm{kg} \mathrm{MS} \cdot \mathrm{ha}^{-1}$ of $B$. brizantha cv. Toledo accumulated according to cutting age and nitrogen fertilization levels, as well as the presence of a possible inflection point, which in the rainy season was located around day 100 and in the dry season around day 40 , associated with the transition between one season and another. Finally, the results suggest that the B. brizantha forage species is influenced by the fertilization dose, age of cut, season and soil and climatic conditions.
\end{abstract}

Keywords: Simulation, script, perennial crops, non-linear models.

\footnotetext{
'Universidad Nacional Experimental de los Llanos Occidentales "Ezequiel Zamora", Carrera 3 Calle 17, Guanare, Portuguesa, Venezuela. ${ }^{2}$ Universidad Nacional Toribio Rodríguez de Mendoza deAmazonas (UNTRM-A), Facultad de Ingeniería Civil y Ambiental, Calle Higos Urco $\mathrm{N}^{\circ}$ 342-350-356, Calle Universitaria N³04, Chachapoyas, Perú

*Autor de correspondencia. E-mail:munuel.milla@untrm.edu.pe
} 


\section{INTRODUCCIÓN}

El crecimiento de los pastos puede ser descrito por medio de funciones matemáticas que predicen el desempeño de la altura y la producción de biomasa. Estas funciones permiten realizar evaluaciones acerca del nivel de producción en las empresas ganaderas y clasificar de forma sencilla la productividad de una especie específica en una zona determinada (Martinez y Herrera, 2007). También permiten calcular los valores máximos y los crecimientos medio y corriente. Con estas funciones se puede además, determinar las edades de corte que permiten obtener el máximo beneficio económico. De igual manera, éstos proveen de información que admite realizar programaciones de alimentación y de capacidad de carga, asimismo permiten medir cambios genéticos de una generación a otra, relacionados con el nivel de producción (Agudelo et al., 2007). En tal sentido, El modelo logístico se encuentra entre las funciones matemáticas que más se han usado con este objetivo. Además de otras, como las lineales y polinómicas (Thornley y France, 2007). Los modelos matemáticos aplicados al crecimiento de las plantas permiten estimar o predecir su comportamiento temporal en diferentes condiciones (Thornley y France, 2007). Para su correcta utilización se deben tener en cuenta tres aspectos fundamentales: a) bondad del ajuste de los datos, b) capacidad de interpretación biológica, c) exigencias computacionales (Chacín, 2000). En la actualidad, este último no constituye una limitación, debido al desarrollo alcanzado por las ciencias computacionales. En ese orden, los programas disponibles para el ajuste de modelos, en especial, los modelos no lineales, entre ellos, el modelo logístico, son de gran utilidad, entre los que destacan el software libre R, el cual fue creado en 1992 en Nueva Zelanda por Ross Ihaka y Robert Gentleman (Ihaka, 1998). Así mismo, R es un lenguaje de programación interpretado, de distribución libre, bajo Licencia GNU, y se mantiene en un ambiente para el cómputo estadístico y gráfico. Este software corre en distintas plataformas Linux, Windows, MacOS, e incluso en PlayStation 3. El término ambiente pretende caracteri- zarlo como un sistema totalmente planificado y coherente, en lugar de una acumulación gradual de herramientas muy específicas y poco flexibles, como suele ser con otro software de análisis de datos. El hecho que $\mathrm{R}$ sea un lenguaje y un sistema, es porque forma parte de la filosofía de creación1, como lo explica John Chambers (Chambers y Hastie, 1991). En tal sentido, el interés de esta investigación se centra en la utilización del entorno de programación del software libre R, con el fin de diseñar un algoritmo que permita simular el comportamiento productivo de pastos, específicamente Brachiaria brizantha $c v$. Toledo bajo fertilización nitrogenada.

\section{METODOLOGÍA}

Los datos se obtuvieron de un ensayo realizado para estudiar el manejo de pastos con fertilización nitrogenada e intervalos de corte en dos suelos del municipio Guanare del estado Portuguesa, en cuyo caso el material vegetal utilizado es semilla de Brachiaria brizant$h a c v$. Toledo con fertilización básica y aplicación en repartida en proporciones iguales, de acuerdo a la distribución de las lluvias (inicio, transición y finales), en abril-mayo, octubre y noviembre-diciembre. Los datos se ajustaron mediante un modelo logístico como se describe a continuación.

La variable respuesta $W(t)$ a considerar para la construcción del modelo (logístico) es la Biomasa Aérea Total, determinada mediante un método destructivo (cuadrícula, estufa), método peso constante. El indicador que describe a esta variable es Kg Materia seca ha1. La variable regresora del modelo $t$ es días de corte y $\beta_{\mathrm{i}}, \mathrm{i}=1,2,3$ los parámetros del modelo.

El análisis estadístico utilizado para la producción de materia seca (MS) acumulada durante las épocas de lluvia y sequía en función de niveles de fertilización nitrogenada e intervalos de corte por finca, fue la suma acumulada de la producción de las fechas de evaluación. Se aplicó el modelo logístico a través de la siguiente fórmula para predecir y ajustar el rendimiento del pasto: 


$$
W(t)=\frac{\beta_{1}}{1+\beta_{2} e^{-\beta_{3} t}}
$$

Donde:

$\mathrm{W}=\mathrm{kg}$ MS acumulada.

$\beta_{1}=\mathrm{kg}$ MS en el día de corte.

$\beta 2=$ constante de integración.

$\beta 3=$ pendiente de la curva (tasa de acumulación de materia seca).

En el modelo se consideró el residual aleatorio con distribución normal e independiente, se utilizó la prueba de Wilk Shapiro. Los análisis se realizaron con el procedimiento $\mathrm{R}$ y se comparó el ajuste de los modelos con base en los criterios siguientes: 1) el error de predicción promedio calculados mediante el coeficiente de determinación múltiple $R 2=(1-(s c e$ $/ s c t)$ ), estimado con base en la suma de cuadrados del error (sce), la suma de cuadrados total corregida (sct), el número de observaciones (n) y el número de parámetros (k) que contiene el modelo.

A continuación se muestra el script en el entorno de programación del software libre R para la estimación de los parámetros del modelo logístico sobre la base de datos de un ensayo en pastos, específicamente $B$. brizantha $c v$. Toledo con fertilización nitrogenada a diferentes edades de corte:

$d c<-c()$

$k g m s<-c()$

K.inicial <- $1.01 * \max (\mathrm{kgms})$

K.inicial

C.inicial <- (K.inicial/kgms[1])-1

C.inicial

N.para.MU.inicial <- kgms[length(kgms)]

N.para.MU.inicial

T.para.MU.inicial <- dc[length $(d c)]$

T.para.MU.inicial

MU.inicial <- $\log ((-$

$1+($ K.inicial/N.para.MU.inicial) $) / C . i n i c i a l)) /(-$

T.para.MU.inicial)

MU.inicial
Datos $<$ - data.frame $(x=d c, y=k g m s)$

fit $<-n l s\left(y \sim K /\left(1+C^{*}\left(\exp \left(-M U^{*} d c\right)\right)\right)\right.$, Datos,

start $=$ list $(K=$ K.inicial, $C=C$.inicial, $M U=$

MU.inicial))

fit

summary(fit)

Coeficientes $<-$ coef(fit)

$K<$ - (as.vector(Coeficientes)) [1]

$C<-($ as.vector(Coeficientes)) [2]

$M U<-($ as.vector(Coeficientes)) [3]

Seguidamente se tiene el script en el entorno de programación del software libre $\mathrm{R}$ para la estimación del coeficiente de determinación del modo logístico con el fin de evaluar la bondad de ajuste de los datos los parámetros del modelo logístico sobre la base de datos de un ensayo en pastos, específicamente $B$. brizantha $c v$. Toledo con fertilización nitrogenada a diferentes edades de corte:

SC.reg $<-$ fit\$m\$deviance()

SC.reg

SC.tot $<-\operatorname{sum}((\mathrm{kgms}$ [1:length(kgms)]mean $(\mathrm{kgms}))^{\wedge} 2$

SC.tot

Sy.x $<-$ summary(fit)\$sigma

Sy.x

R.cuadrada $<-1-(S C . r e g / S C . t o t)$

R.cuadrada

Finalmente, se presenta el script en el entorno de programación del software libre $\mathrm{R}$ para evaluar gráficamente la bondad de ajuste de los datos mediante la construcción de bandas de confianza del modelo logístico:

$\operatorname{Logistica}<-$ function $(x)\left\{K /\left(1+C^{*}\left(\exp \left(-M U^{*} x\right)\right)\right)\right\}$

$\operatorname{plot}(x=d c, y=k g m s)$

points $(x=c(0: 378)$, Logistica $(c(0: 378))$, type $=" l ")$

se.fit.confianza $<-$

sqrt(apply(fit\$m\$gradient(), 1, function(x)sum(vco

$v(f i t) * \operatorname{outer}(x, x))))$ 


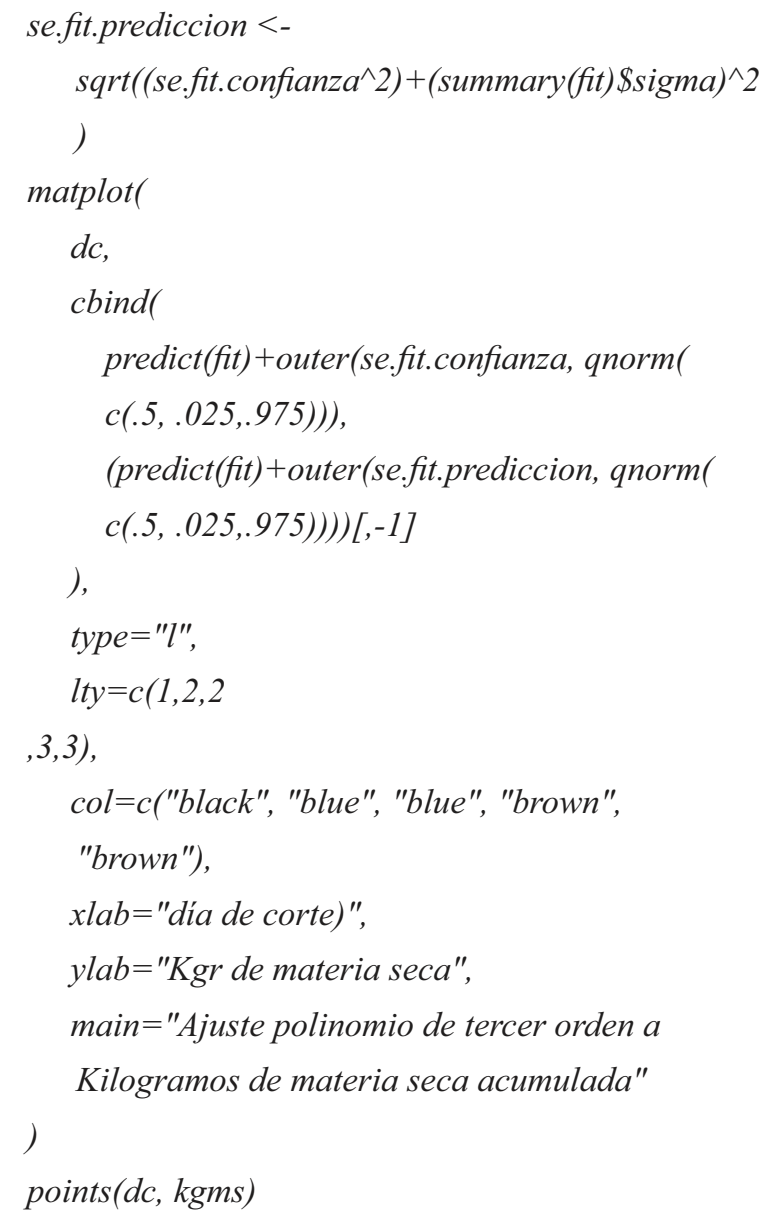

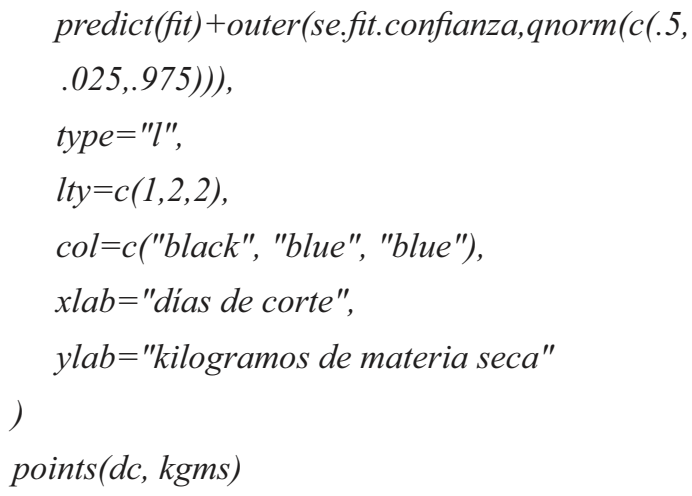

A continuación se muestra el script en el entorno de programación del software libre R para la estimación de los parámetros del modelo logístico sobre la base de datos de un ensayo en pastos, específicamente B. brizantha cv. Toledo con fertilización nitrogenada a diferentes edades de corte:

$d c<-c()$

kgms $<-c()$

K.inicial $<-1.01 *^{\max }(\mathrm{kgms})$

K.inicial

C.inicial <- (K.inicial/kgms[1])-1

C.inicial

N.para.MU.inicial <- kgms[length(kgms)]

N.para.MU.inicial

T.para.MU.inicial <- dc[length $(d c)]$

T.para.MU.inicial

MU.inicial <- $(\log ((-$

$1+($ K.inicial/N.para.MU.inicial))/C.inicial) $) /(-$

T.para.MU.inicial)

MU.inicial

Datos $<$ - data.frame $(x=d c, y=k g m s)$

fit $<-n l s\left(y \sim K /\left(1+C^{*}\left(\exp \left(-M U^{*} d c\right)\right)\right)\right.$, Datos, start $=$ list $(K=K$.inicial, $C=C$.inicial, $M U=$ MU.inicial))

fit

summary(fit)

Coeficientes $<-$ coef(fit)

$K<$-(as.vector(Coeficientes)) [1]

$C<-($ as.vector(Coeficientes)) [2]

$M U<-($ as.vector(Coeficientes) $)[3]$

Seguidamente se tiene el script en el entorno de programación del software libre $\mathrm{R}$ para la estimación 
del coeficiente de determinación del modo logístico con el fin de evaluar la bondad de ajuste de los datos los parámetros del modelo logístico sobre la base de datos de un ensayo en pastos, específicamente B. brizantha cv. Toledo con fertilización nitrogenada a diferentes edades de corte:

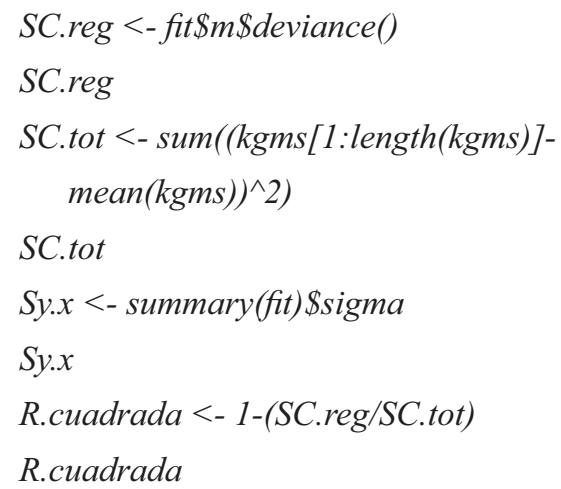

"brown"),

$x l a b=$ "día de corte)",

$y l a b=" K g r$ de materia seca",

main="Ajuste polinomio de tercer orden a

Kilogramos de materia seca acumulada"

)

points (dc, kgms)

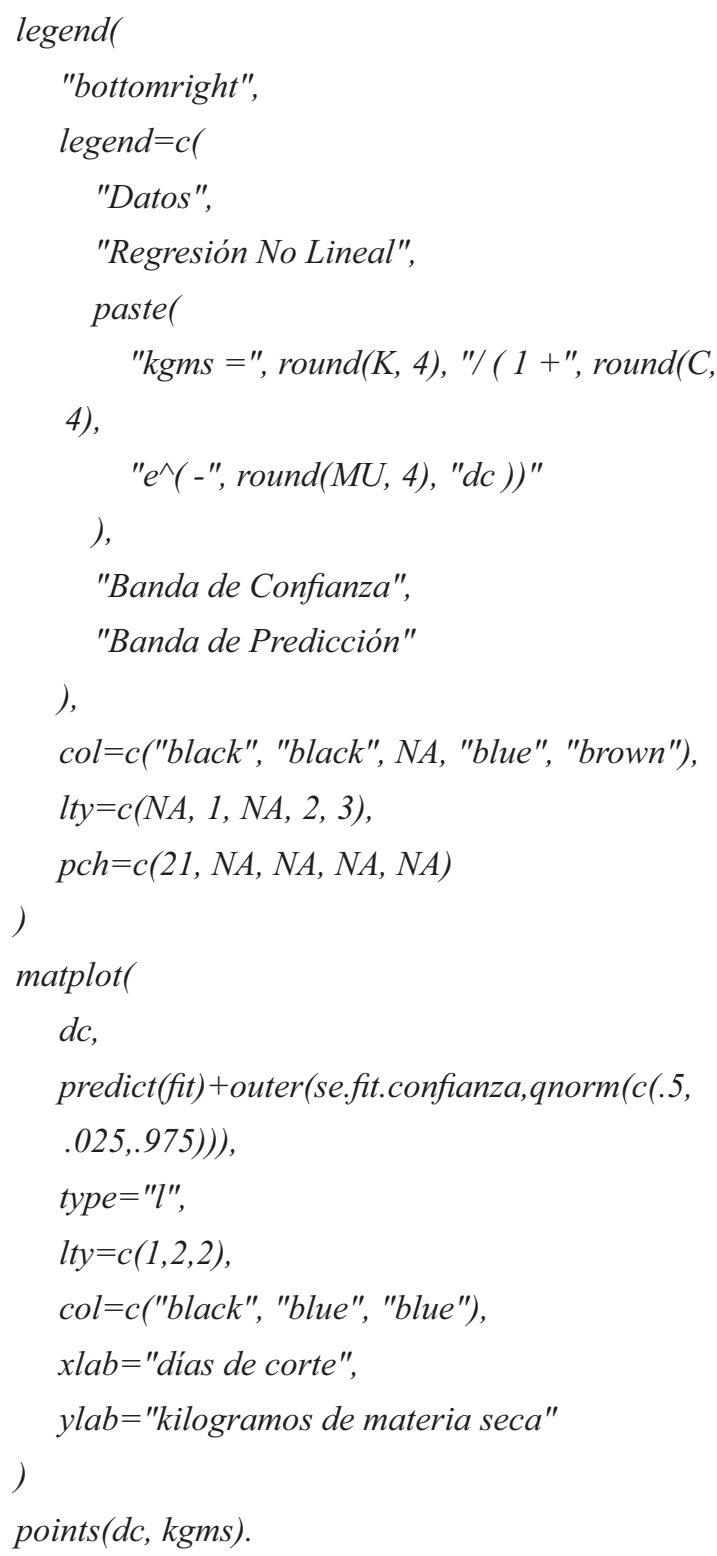

\section{RESULTADOS Y DISCUSIÓN}

En la Tabla 1 se muestran los resultados de la estimación de los parámetros del modelo logístico, donde los $\mathrm{kg} \mathrm{MS} \cdot \mathrm{ha}^{-1}$ acumulados en el día de corte $(\mathrm{k})$ aumentaron al incrementar la edad y niveles de fertilización 
Tabla 1. Parámetros de un modelo logístico para estimar la producción ( $\mathrm{kg} \mathrm{MS*ha-1)} \mathrm{de} B$. brizantha cv. Toledo en función de los días de corte y fertilización nitrogenada en épocas de lluvia y sequía.

\begin{tabular}{|c|c|c|c|c|c|c|}
\hline Época & Edad & $\operatorname{kgN} N \cdot h a-1 \cdot a \tilde{n o-1}$ & 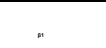 & $\beta 2$ & $\beta 3$ & $\mathbf{R 2}$ \\
\hline \multirow{16}{*}{ Lluvia } & \multirow{4}{*}{21} & 0 & 8107 & 7,8 & 0,03 & 0,98 \\
\hline & & 30 & 11080 & 10,4 & 0,02 & 0,99 \\
\hline & & 60 & 12300 & 12,8 & 0,02 & 0,97 \\
\hline & & 120 & 12580 & 9,21 & 0,02 & 0,97 \\
\hline & \multirow{4}{*}{28} & 0 & 18690 & 4,3 & 0,02 & 0,95 \\
\hline & & 30 & 23750 & 7,3 & 0,01 & 0,98 \\
\hline & & 60 & 25250 & 5,7 & 0,01 & 0,98 \\
\hline & & 120 & 25870 & 4,9 & 0,02 & 0,96 \\
\hline & \multirow{4}{*}{35} & 0 & 21450 & 5,2 & 0,02 & 0,95 \\
\hline & & 30 & 27950 & 6 & 0,02 & 0,97 \\
\hline & & 60 & 28190 & 5,5 & 0,02 & 0,97 \\
\hline & & 120 & 29570 & 4,9 & 0,02 & 0,96 \\
\hline & \multirow{4}{*}{42} & 0 & 26580 & 6,8 & 0,02 & 0,99 \\
\hline & & 30 & 29730 & 6,5 & 0,02 & 0,98 \\
\hline & & 60 & 33640 & 5,2 & 0,02 & 0,98 \\
\hline & & 120 & 38420 & 5,9 & 0,02 & 0,98 \\
\hline \multirow{16}{*}{ Sequía } & \multirow{4}{*}{21} & 0 & 1658 & 35,3 & 0,06 & 0,99 \\
\hline & & 30 & 1856 & 12,25 & 0 & 0,91 \\
\hline & & 60 & 2430 & 27 & 0,06 & 0,99 \\
\hline & & 120 & 2759 & 24,8 & 0,06 & 0,99 \\
\hline & \multirow{4}{*}{28} & 0 & 8875 & 14,2 & 0,04 & 0,99 \\
\hline & & 30 & 2760 & 10 & 0,02 & 0,99 \\
\hline & & 60 & 10990 & 11,4 & 0,04 & 0,99 \\
\hline & & 120 & 11890 & 10,9 & 0,03 & 0,99 \\
\hline & \multirow{4}{*}{35} & 0 & 9504 & 15 & 0,04 & 0,99 \\
\hline & & 30 & 10590 & 25,4 & 0,05 & 0,99 \\
\hline & & 60 & 12700 & 12,8 & 0,04 & 0,99 \\
\hline & & 120 & 13770 & 19,7 & 0,04 & 0,99 \\
\hline & \multirow{4}{*}{42} & 0 & 12210 & 7,9 & 0,03 & 0,97 \\
\hline & & 30 & 16920 & 11,3 & 0,02 & 0,99 \\
\hline & & 60 & 17030 & 7,4 & 0,03 & 0,99 \\
\hline & & 120 & 20670 & 6,7 & 0,03 & 0,99 \\
\hline
\end{tabular}

nitrogenada en amabas épocas. El modelo se caracteriza por formar una curva sigmoidea que se ajusta al crecimiento típico de las plantas (Pérez-Amaro et al., 2004; Thornley y France, 2007). Así pues, esta clase de modelos es utilizada en diferentes áreas y constituyen una herramienta de gran aplicación en el estudio de sistemas con diversas características (Torres et al., 2009). De la misma manera, el modelo logístico se encuentra entre los más utilizados en el campo de la ciencia animal para describir el crecimiento de individuos, y además es capaz de identificar parámetros productivos de interés económico que involucran la implementación de pro- gramas de mejoramiento genético (Cayré et al., 2007; Casas et al., 2010; Martinez y Herrera, 2007). En ese orden, en investigaciones hechas por (Machado et al., 1983) se verifica que el modelo logístico muestra un buen ajuste en relación a datos experimentales de rendimiento y altura de otras especies y variedades de pastos, entre los que destacan Panicum maximun vc. Mombaza y Pennisetum purpureum vc. Cuba CT-115, las cuales se caracterizan por ser variedades con un ciclo largo de más de 160 días. En ambas épocas del año, los parámetros fueron significativos, y los coeficientes de determinación mayores a 91\%, lo que sugiere que el modelo 
logístico muestra una alta precisión para explicar los $\mathrm{kg}$ MS $\cdot h^{-1}$ de $B$. brizantha $c v$. Toledo acumulados en función de la edad de corte y de los niveles de fertilización nitrogenada en amabas épocas, lo cual se evidencia en las Figuras 1 y 2 , en donde se observa la presencia de un posible punto de inflexión, el cual en la época de lluvia
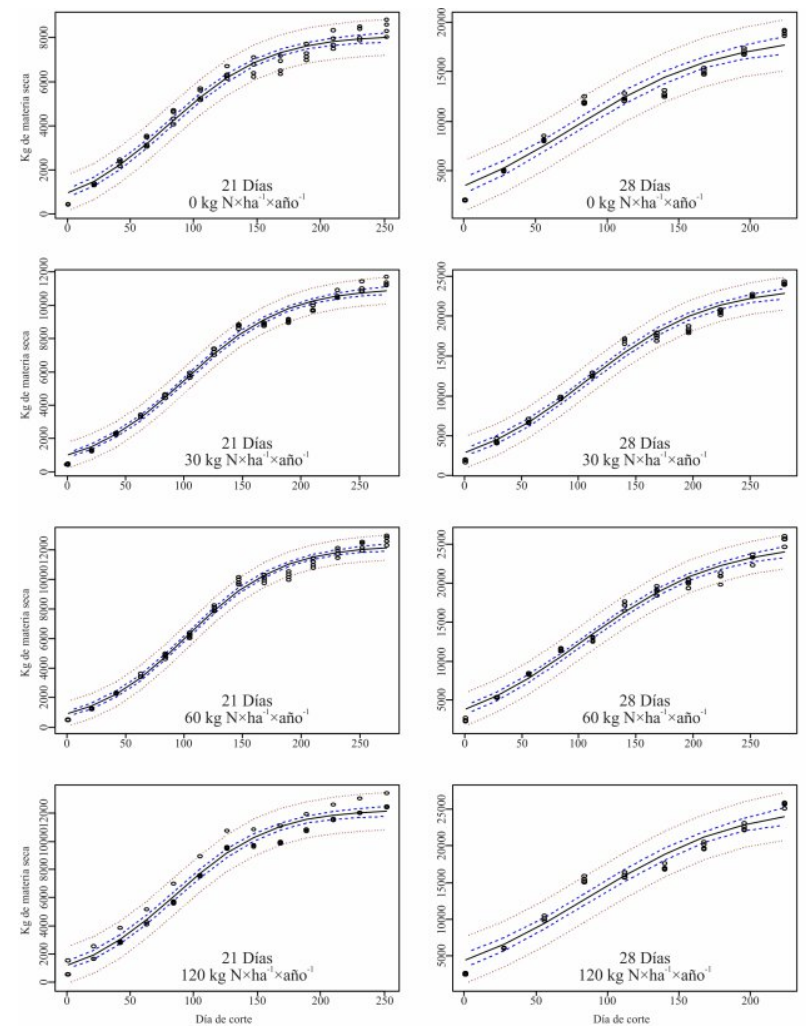

se ubica alrededor del día 100 y en la época de sequía alrededor del día 40. Así mismo, se observa una mayor variabilidad en el período lluvioso con respecto al seco para la acumulación de materia seca, este comportamiento puede obedecer a que en el período lluvioso existe mayor velocidad de crecimiento.
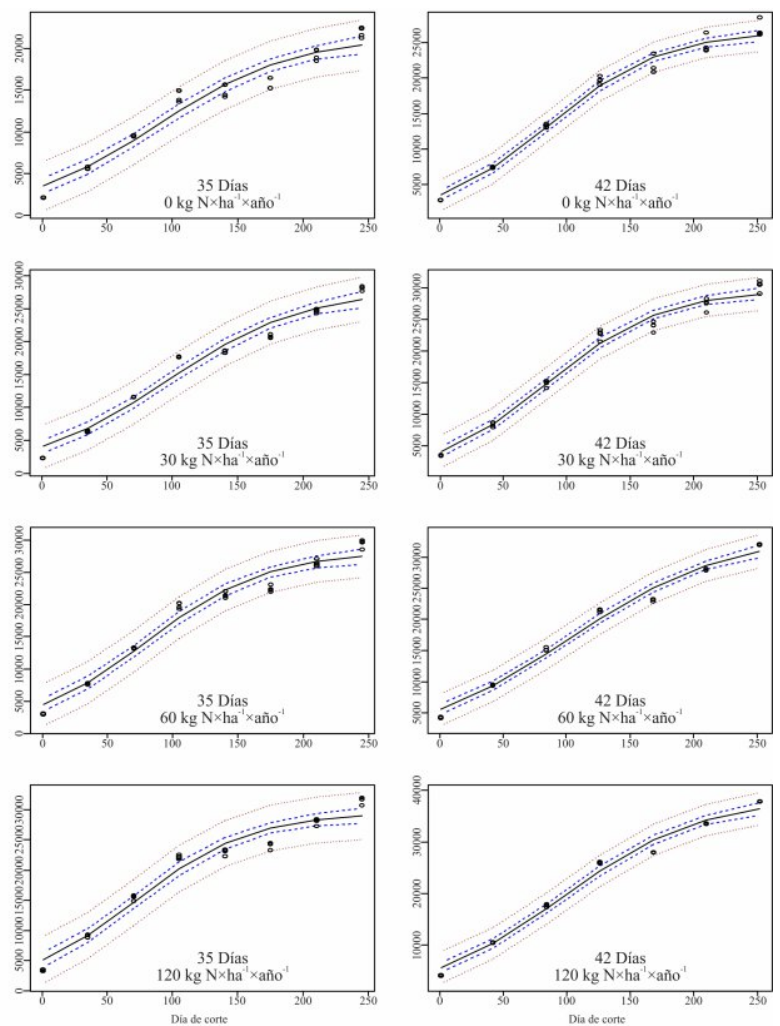

Figura 1. Curvas de rendimiento $\left(\mathrm{kg} \mathrm{MS} \cdot \mathrm{ha}^{-1}\right)$ de $B$. brizantha $\mathrm{cv}$. Toledo en época de lluvia a través del modelo Logístico.

El análisis de varianza mostró diferencias en el rendimiento de materia seca del pasto $B$. brizantha $\mathrm{cv}$. Toledo en ambas épocas $(\mathrm{P}<0,01)$. Así mismo, la materia seca acumulada fue influenciada por la dosis de nitrógeno, reportándose el mayor rendimiento acumulado de $120 \mathrm{~kg} \mathrm{~kg} \cdot \mathrm{ha}^{-1} \cdot \mathrm{año}^{-1}$ por época (Tabla 2). En la época de lluvia el rendimiento fue mayor $(22130 \mathrm{~kg}$ $\left.\mathrm{MS} \cdot \mathrm{ha}^{-1}\right)$ en comparación con la época seca $(8775 \mathrm{~kg}$ $\mathrm{MS} \cdot \mathrm{ha}^{-1}$ ). Estos resultados concuerdan con (Martuscello et al., 2009) quienes señalaron que en Brachiaria cv. Xaraés se encontró que por cada kilogramo de nitrógeno aplicado se alcanzó un aumento de 5,6 $\mathrm{kg} \cdot \mathrm{ha}^{-1}$ materia seca en la época de lluvia. Así mismo, el rendimiento en materia seca acumulada se incrementó a medida que la dosis de nitrógeno fue mayor, alcanzando su máximo en $120 \mathrm{~kg} \cdot \mathrm{ha}^{-1} \cdot \mathrm{año}^{-1}$.
En el Tabla 3 se observa como el rendimiento de materia seca incrementó a medida que aumentaron los intervalos de corte en ambas épocas $(\mathrm{P}<0,01)$. El mayor rendimiento se presentó con 42 días entre corte en la época de lluvia y sequía (30345 y 12731 kg $\mathrm{MS} \cdot \mathrm{ha}^{-1}$, respectivamente). Para la época de sequía la producción de la MS registró los valores más altos a los 42 días, que sobrepasaron las treinta toneladas en el año. Esto coincide con lo señalado por (Machado et al., 1983), quien sugiere que en la mayoría de los ensayos con gramíneas tropicales, las frecuencias de corte más prolongadas, sin que se perjudique la calidad del pasto, son las más favorables y proporcionan mayor estabilidad al pastizal. De esta manera, el incremento del rendimiento con la edad hasta los 42 días pudo deberse, principalmente, al proceso fotosintético que 

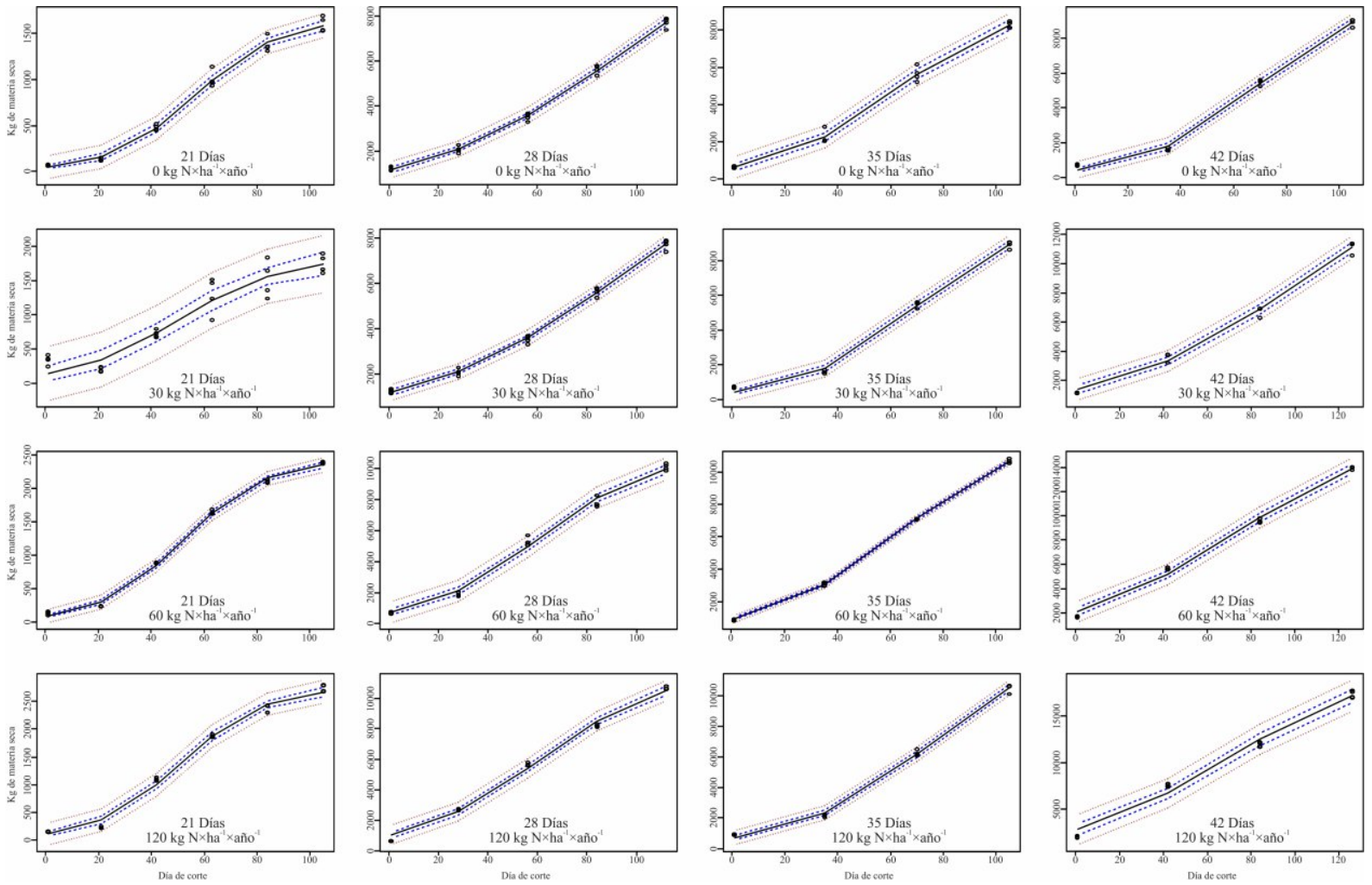

Figura 2. Curvas de rendimiento $\left(\mathrm{kg} \mathrm{MS} \cdot \mathrm{ha}^{-1}\right)$ de $B$. brizantha $\mathrm{cv}$. Toledo en época de sequía a través del modelo Logístico.

Tabla 2. Rendimiento $\mathrm{kg} \mathrm{MS} \cdot \mathrm{ha}^{-1}$ del pasto B. brizantha $\mathrm{cv}$. Toledo en función de la fertilización nitrogenada.

\begin{tabular}{cccc}
\hline Nitrógeno $\mathbf{k g} \cdot \mathbf{h a}^{\mathbf{1}} \cdot \mathbf{a n ̃ o}^{-\mathbf{1}}$ & Lluvia & Sequía & TotalAnual \\
\hline 0 & $18010 \mathrm{a}$ & $6694 \mathrm{a}$ & 24979 \\
30 & $21904 \mathrm{~b}$ & $7840 \mathrm{~b}$ & 29744 \\
60 & $23195 \mathrm{c}$ & $9565 \mathrm{c}$ & 32760 \\
120 & $25411 \mathrm{~d}$ & $10724 \mathrm{~d}$ & 36135 \\
\hline Promedio & $\mathbf{2 2 1 3 0}$ & $\mathbf{8 7 7 5}$ & $\mathbf{3 0 9 0 5}$ \\
\hline
\end{tabular}

Tabla 3. Rendimiento $\mathrm{kg} \mathrm{MS} \cdot \mathrm{ha}^{-1} \mathrm{del}$ pasto B. brizantha $\mathrm{cv}$. Toledo en función de los intervalos de corte.

\begin{tabular}{cccc}
\hline \multirow{2}{*}{ IC días } & \multicolumn{3}{c}{${\text { Rendimiento, } \mathbf{k g} \text { MS } \cdot \mathbf{h a}^{\mathbf{1}}}$} \\
\cline { 2 - 4 } & Lluvia & Sequía & TotalAnual \\
\hline 21 & $10651 \mathrm{~d}$ & $2147 \mathrm{~d}$ & 12798 \\
28 & $21817 \mathrm{c}$ & $9571 \mathrm{c}$ & 31387 \\
35 & $25707 \mathrm{~b}$ & $10651 \mathrm{~b}$ & 36358 \\
42 & $30345 \mathrm{a}$ & $12731 \mathrm{a}$ & 43077 \\
\hline Promedio & $\mathbf{2 2 1 3 0}$ & $\mathbf{8 7 7 5}$ & $\mathbf{3 0 9 0 5}$ \\
\hline
\end{tabular}

suministra las sustancias y energías necesarias para el crecimiento y desarrollo de la planta (Herrera and Ramos 2006), lo que provoca la acumulación de materia seca. El rendimiento de materia seca en el período lluvioso fue 3,8 y 2,5 veces superior con relación a la sequía a los 42 días. De allí que, Esto corrobora las dificultades que se presentan en la producción de forraje, que están motivadas por bajas temperaturas, poca humedad en el suelo y duración del día. La espe- cie forrajera $B$. brizantha $\mathrm{cv}$. Toledo presenta una marcada estacionalidad en el rendimiento en la época seca.

El efecto de la interacción entre la fertilización nitrogenada y la edad de corte sobre el rendimiento de materia seca en la época de lluvia $(\mathrm{P}<0,01)$ se presenta en la Figura 3, allí se observa como la producción de materia seca de esta especie de pasto depende de la cantidad de nitrógeno aplicada y de la edad de corte. El gráfico 
muestra como a medida que se incrementaron los intervalos de corte y la fertilización nitrogenada la producción de materia seca también se icrementó. El mayor rendimiento correspondió con 42 días de corte y $120 \mathrm{~kg}$ $\mathrm{N} /$ ha en comparación con el testigo 35884 y $25844 \mathrm{~kg}$ $\mathrm{MS} \cdot \mathrm{ha}^{-1}$, lo que significa un aumento de 32,3 y $28,0 \%$ en relación al tratamiento sin fertilización. Estos resultados muestran que la especie forrajera responde a la fertilización nitrogenada. Estos datos coinciden con Rodríguez et al. (2011), que encontró que la fertilización nitrogenada tiene un efecto positivo sobre la producción de materia seca total de $B$. brizantha.

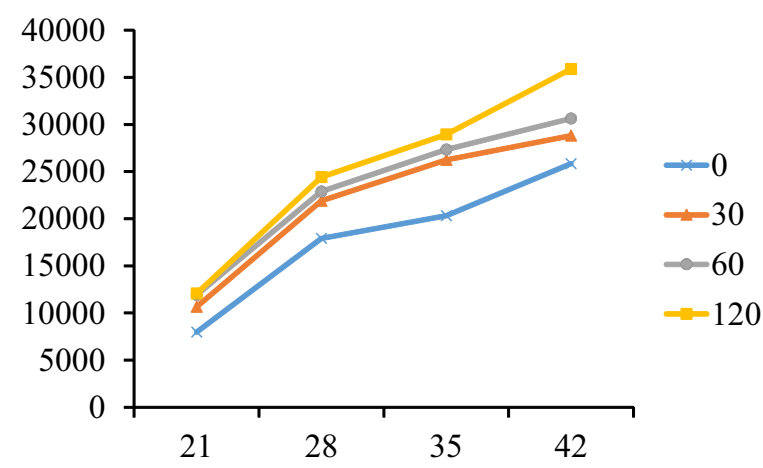

Figura 3. Rendimiento $\mathrm{kg} \mathrm{MS} \cdot \mathrm{ha}^{-1}$ del pasto $B$. brizantha $\mathrm{cv}$. Toledo en función de la fertilización nitrogenada y edades de corte en la época lluviosa.

En la Figura 4 se presenta la interacción de la fertilización por edad de corte en la especie forrajera $B$. brizantha durante la época seca $(\mathrm{P}<0,01)$. Allí se muestra como existe una influencia de la fertilización nitrogenada y la edad de corte en el rendimiento de la producción de materia seca. La mayor producción fue con la dosis $120 \mathrm{~kg} \mathrm{~N} / \mathrm{ha}$ y 42 días de corte $(17084 \mathrm{~kg}$ $\left.\mathrm{MS} \cdot \mathrm{ha}^{-1}\right)$. Se observa que el rendimiento se incrementó a los 35 días y 42 días de corte con $60 \mathrm{~kg} \mathrm{~N} / \mathrm{ha}(2748$ y $\left.3753 \mathrm{~kg} \mathrm{MS} \cdot \mathrm{ha}^{-1}\right)$ y $120 \mathrm{~kg} \mathrm{~N} / \mathrm{ha}(2880$ y $4249 \mathrm{~kg}$ $\left.\mathrm{MS} \cdot \mathrm{ha}^{-1}\right)$. Estos resultados sugieren que la especie forrajera B. brizantha está influenciada por las dosis de fertilización, edad de corte, época del año y condiciones edafoclimáticas.

\section{CONCLUSIONES Y RECOMENDACIONES}

Los resultados de esta investigación en primera instancia evidencian la utilidad del software R en la construcción de bandas de confianza sobre el ajuste de modelos no lineales, específicamente el logístico para

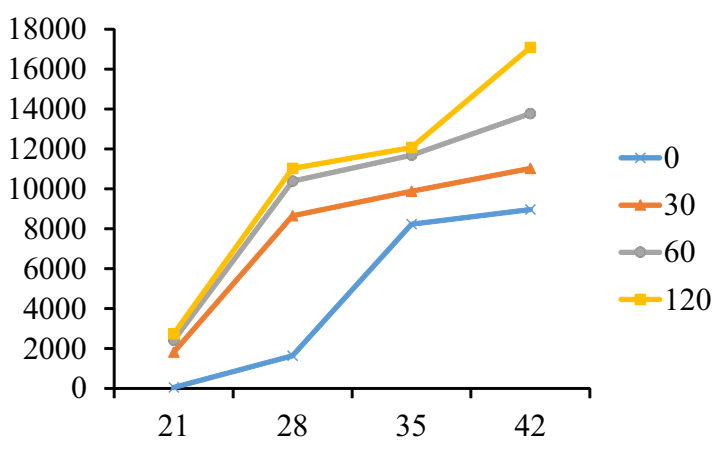

Figura 4. Rendimiento $\mathrm{kg} \mathrm{MS} \cdot \mathrm{ha}^{-1} \mathrm{del}$ pasto $B$. brizantha $\mathrm{cv}$. Toledo en función de la fertilización nitrogenada y edades de corte en la época seca.

la estimación de la producción de materia seca en pastos bajo condiciones de fertilización nitrogenada a diferentes edades de corte. De la misma manera, los datos experimentales se ajustaron a un modelo caracterizado por formar una curva sigmoidea típica del crecimiento de las plantas.

En ambas épocas del año el modelo logístico mostró una alta precisión para explicar los $\mathrm{kg} \mathrm{MS} \cdot \mathrm{ha}^{-1}$ de $B$. brizantha $c v$. Toledo acumulados en función de la edad de corte y de los niveles de fertilización nitrogenada, así como la presencia de un posible punto de inflexión, el cual en la época de lluvia se ubicó alrededor del día 100 y en la época seca alrededor del día 40, asociado a la transición entre una época y otra.

Así mismo, se observó una mayor variabilidad en el período lluvioso con respecto al seco para la acumulación de materia seca, asociada principalmente a la alta tasa de crecimiento ocurrida en la época de lluvia.

Estos resultados sugieren que la especie forrajera $B$. brizantha está influenciada por la dosis de fertilización, edad de corte, época del año y las condiciones edafoclimáticas.

Finalmente, en virtud de los resultados obtenidos en esta investigación se recomienda el uso del software $\mathrm{R}$, específicamente para el ajuste de modelos de crecimiento asociados a datos provenientes de ensayos de fertilización nitrogenada a distintas edades de corte para estimar la producción de materia seca en pastos. Así mismo, se sugiere calcular la primera y segunda derivada del modelo para determinar la máxima velocidad de crecimiento y edad a la que se alcanza el 
punto de inflexión. De igual manera se aconseja probar otros modelos no lineales aplicados en la dinámica del crecimiento de los pastos tales como Gompertz y exponencial.

\section{REFERENCIAS BIBLIOGRÁFICAS}

Agudelo, D., M. Cerón, y L. Restrepo. 2007. “Modelación de Las Funciones de Crecimiento Aplicadas a La Producción Animal." Revista Colombiana de Ciencias Pecuarias 20: 157-73.

Casas, G. A., D. Rodríguez, y G. A. Téllez. 2010. "Propiedades Matemáticas Del Modelo de Gompertz y Su Aplicación Al Crecimiento de Los Cerdos." Revista Colombiana de Ciencias P ecuarias $23: 349-58$. doi:10.5944/educxx1.17.1.10708.

Cayré, M. E., G. M. Vignolo, y O. A. Garro. 2007. "Selección de Un Modelo Primario Para Describir La Curva de Crecimiento de Bacterias Lácticas y Brochothrix Thermosphacta Sobre Emulsiones Cárnicas Cocidas.” Informacion Tecnologica 18 (3): 23-29. doi:10.4067/S0718-07642007000300004.

Chacín, F. 2000. Diseño y Análisis de Experimentos. Caracas (Venezuela): Universidad Central de Venezuela.

Chambers, J. M., y T. J: Hastie. 1991. Statistical Models in S. Seattle (EEUU): Wadsworth \& Brooks/Cole Advanced Books \& Software.

Herrera, R. S., y N. Ramos. 2006. "Evaluación Agronómica y Calidad.” En Pennisetum Purpureum Para La Ganaderia Tropical. Herrera, R.S., G. Crespo, y G. Febles (eds). La Habanan (Cuba): EDICA.

Ihaka, R. 1998. "R: Past and Future History." En $X X X$ Symposium on the Interface. Quebec (Cana$\mathrm{d}$ a ). h t t p : / / c r a n . r project.org/doc/html/interface 98 paper/paper.html.

Machado, R., O. Cáceres, and R. Miret. 1983. "Pennisetum Purpureum Cvs. Taiwan A-144 A-146 A-148 y 801-4.” Pastos y Forrajes 6
(2): 143-59. http://www.sidalc.net/cgibin/wxis.exe/?IsisScript=catalco.xis\&metho $\mathrm{d}=$ post \&formato $=2 \&$ cantidad $=1 \&$ expresion $=\mathrm{mfn}=053498$.

Martinez, R. O., y R.S. Herrera. 2007. “Un Modelo De Manejo Del Pasto En El Periodo Seco Para La Producción De Leche." En XI Seminario Manejo y Utilizaciòn de Pastos y Forrajes En Sistemas de Producciòn Animal. La Habana (Cuba).

Martuscello, J. A., L. Jank, M. Marques, G. Neto, A. Laura, D. Noronha, and F. Vieira. 2009. "Produção de Gramíneas Do Gênero Brachiaria Sob Níveis de Sombreamento." Revista Brasileira de Zootecnia 38 (7): 1183-90.

Pérez-Amaro, J. A., E. G. Moya, J. F. E. Quiroz, A. R. Q. Carrillo, J. Pérez-Pérez, y A. HernándezGaray. 2004. "Análisis de Crecimiento, Área Foliar Específica y Concentración de Nitrógeno En Hojas de Pasto 'Mulato' (Brachiaria Híbrido, Cv.).” Técnica Pecuaria En México 42 (3): 447-58.

Rodríguez, L., V. Torres, R. O. Martínez, O. Jay, A. C. Noda, y M. Herrera. 2011. "Models to Estimate the Growth Dynamics of Pennisetum purpureum cv. Cuba CT-169." Cuban Journal of Agricultural Science 45 (4): 349-54.

Thornley, J. H. M., y J. France. 2007. Mathematical Models in Agriculture: Quantitative Methods for the Plant, Animal and Ecological Sciences. Londre (Reino Unido): Cromwell Press.

Torres, V., I. Sampaio, y O. Fundora. 2009. “Modelo Estocástico Del Comportamiento Productivo En La Etapa Crecimiento de Búfalos de Agua En Cuba." Revista Cubana de Ciencia Agrícola 43 (2): 113-17. 